\title{
Eine Hall-Generatoranordnung zur Messung kleiner Temperaturdifferenzen an Metallen
}

\author{
Von A. J. Schmidt \\ Aus dem Elektrotechnischen Institut der Technischen Hochschule, Wien \\ (Z. Naturforschg. 18 a, 37-38 [1963] ; eingegangen am 11. Oktober 1962)
}

\begin{abstract}
Eine Anordnung zur Messung von Temperaturdifferenzen an kleinen metallischen Proben wird beschrieben. Ein Auftrennen des Thermokreises in zwei galvanisch getrennte ermöglicht den metallischen Kontakt beider Temperaturfühler mit der Probe. Die beiden Teilspannungen werden mit Hilfe eines HALL-Generators voneinander subtrahiert.
\end{abstract}

Zum Studium des Einflusses des Magnetfeldes auf die Thermokraft von Ferromagneticis - über die Ergebnisse wird in ${ }^{1}$ berichtet - war es notwendig, an kleinen Metallstäbchen $\left(13 \times 1 \times 1 \mathrm{~mm}^{3}\right)$ Temperaturdifferenzen von nur wenigen Graden auf mindestens 5\% konstant zu halten und zu messen. Eine Messung mit einem Differentialthermoelement stößt auf erhebliche Schwierigkeiten, da einerseits die Temperaturfühler in gutem thermischem Kontakt mit der Probe sein sollen, andererseits elektrisch von ihr isoliert sein müssen. Die Situation ist in Abb. 1 schematisch skizziert; Abb. 2 zeigt dazu ein „Ersatzschaltbild“, wobei R der Wärmewiderstand der Probe, $R_{2}$ und $R_{3}$ die Übergangswiderstände zwischen den Lötstellen A und D bzw. B und C sind. Die Wärmeleitung über das Thermoelement zwischen $\mathrm{A}$ und $\mathrm{B}$ ist durch $\mathrm{R}_{1}$ charakterisiert. $\Delta T$ ist die an der Probe wirksame, $\Delta T^{\prime}$ die gemessene Temperaturdifferenz. Man erkennt unschwer, daß die beiden um so besser übereinstimmen, je kleiner der Bruch $\left(R_{2}+R_{3}\right) / R_{1}$ ist. Einer Vergrößerung von $R_{1}$ sind sehr bald Grenzen gesetzt, da man aus experimentellen Gründen nicht beliebig dünne Drähte für die Thermoelemente verwenden kann und außerdem A und $\mathrm{B}$ nur geringen Abstand voneinander haben. Trennt man hingegen den Thermokreis in zwei Teile auf (Abb. 1 b), so erhält man die Möglichkeit, A und $\mathrm{D}$ bzw. $\mathrm{B}$ und $\mathrm{C}$ zu verlöten und so die thermischen Übergangswiderstände zu vermeiden. Eine getrennte Messung der beiden Thermospannungen und nachträgliche Differenzbildung ist nicht nur umständlich, sondern reduziert, für den Fall, daß $\Delta T<T-T_{\mathrm{v}}$ ist, die Genauigkeit der $\Delta T$-Messung beträchtlich, so daß eine Subtraktion auf elektrischem Weg zweckmäßig erscheint. Da die Verwendung eines Galvano1 A. J. Sснмidt u. K. M. Косн, Z. Naturforschg. 18 a, 31
[1963]. meters mit zwei gleichen, galvanisch getrennten Wicklungen auf Grund der Kosten eines derartigen Spezialgeräts erschwert ist, beschritten wir einen anderen Weg. Die beiden zu subtrahierenden Spannungen wurden in passender Polung an zwei gleich

Abb. 1. Zur Temperaturmessung an der Probe, a) mit einem Thermokreis, b) mit zwei getrennten;

$T_{\mathrm{V}}$ Temperatur der Vergleichslötstelle, 1 Probe, 2, 3 Kupferanschlüsse.

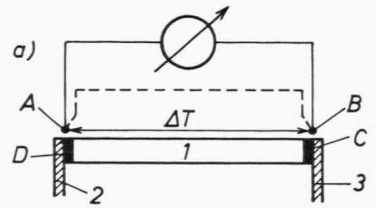

Abb. 2. Ersatzschaltbild

für die Temperaturmessung.
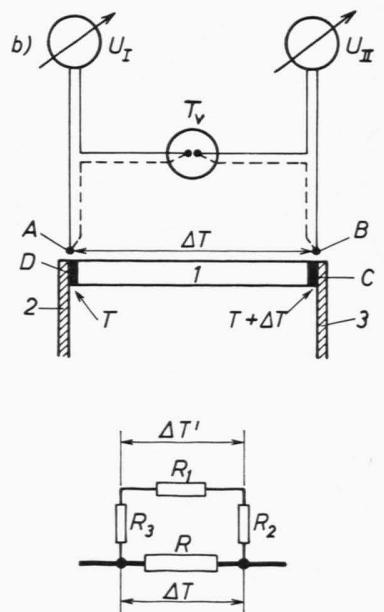

$$
\Delta T^{\prime} \rightarrow \Delta T \text { wenn } \frac{R_{2}+R_{3}}{R_{1}} \rightarrow 0
$$

große galvanisch getrennte Feldwicklungen eines HaLL-Generators ${ }^{2}$ gelegt. Die HaLL-Spannung $U_{\mathrm{h}}$ ist proportional dem magnetischen Feld, dieses der Differenz der Ströme $I_{\mathrm{I}}$ und $I_{\mathrm{II}}$, diese wiederum den angelegten Spannungen $U_{\mathrm{I}}$ und $U_{\mathrm{II}} ; U_{\mathrm{h}}$ ist daher letzten Endes direkt proportional der Temperatur-

\footnotetext{
2 Von den am Markt befindlichen Typen ist der Hall-Generator SBV 514 am besten geeignet.
} 
differenz. Für den sekundär unbelasteten HaLLMultiplikator gilt

$U_{\mathrm{h}}=\left(R_{\mathrm{h}} i_{\mathrm{s}} B\right) / d$, wobei $B=w i_{\mathrm{f}} /\left(\delta+l_{\mathrm{Fe}} / \mu_{\mathrm{a}}\right)$ ist.

Dabei bedeuten: $R_{\mathrm{h}}$ HaLL-Konstante, $i_{\mathrm{S}}$ Steuerstrom, $d$ Dicke des HalL-Plättchens, $w$ Windungszahl, $\mu_{\mathrm{a}}$ Anfangspermeabilität des Kerns, $\delta$ Luftspalt des Kerns, $l_{\mathrm{Fe}}$ Länge der Kraftlinien im Kern, $R_{\mathrm{i}}$ Widerstand des Thermoelements, $R_{\mathrm{w}}$ Widerstand der Wicklung.

Bis auf das Produkt $w i_{\mathrm{f}}$ ist $U_{\mathrm{h}}$ durch die Wahl des Typs des Hall-Multiplikators ${ }^{3}$ bestimmt. Aus der Bedingung

$$
w i_{\mathrm{f}}=\max
$$

folgt durch einfache Rechnung $R_{\mathrm{i}}=R_{\mathrm{w}}$.

Diese Überlegungen ergaben unter Berücksichtigung der von uns verwendeten Thermoelemente $\left(R_{\mathrm{i}}=0,2 \Omega\right)$ für jede der beiden Wicklungen $R_{\mathrm{w}}=0,2 \Omega$ und $w=49$. Die Bewicklung wurde bifilar aufgebracht.

Um die Hall-Anordnung zur Messung der Temperaturdifferenz zu eichen, wurde an Stelle der Probe ein Konstantandraht mit genau bekannter Thermokraft in die Halterung eingelötet. An den Anschlüssen 2 und 3, an denen sonst die Änderung der Thermokraft gemessen wurde, maßen wir über den neugebildeten $\mathrm{Cu}-$ Konstantankreis die Temperaturdifferenz der beiden Lötstellen und setzten sie mit der am Ausgang des HALL-Generators auftretenden Spannung in Beziehung.

\footnotetext{
${ }^{3}$ F. Kunrt, ETZ-A 78, 342 [1957].
}

Um die OHmsche Restkomponente möglichst einfach abgleichen zu können, ist eine der beiden HaLLElektroden doppelt nach außen geführt (Abb. 3). Das Potentiometer P dient allerdings nur der Grobeinstellung. Der Feinabgleich erfolgt mit Hilfe eines in der Nähe des HaLL-Generators befindlichen drehbaren Permanentmagneten.

Abb. 3.

Der HALL-Generator.

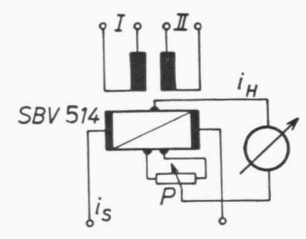

Als Anzeigeinstrument wurde ein Lichtmarkeninstrument $\left(3 \cdot 10^{-7} \mathrm{~V} / \mathrm{Skt}, R_{\mathrm{i}}=10 \Omega\right)$ verwendet. Die Gesamtempfindlichkeit der Anlage beträgt für einen Steuerstrom von $400 \mathrm{~mA}$ 94,6 Tlst/Grad. Das bedeutet, daß $0,01^{\circ}$ noch abgelesen werden können.

Die beiden Hälften der Bewicklung sind im Rahmen der Meßgenauigkeit als gleich zu betrachten (1\%). Wir überzeugten uns experimentell von der Linearität des Zusammenhangs von $\Delta T$ und $U_{\mathrm{h}}$; die Abweichungen waren $<1 \%$.

Die zeitliche Konstanz der Anlage ist befriedigend. Der Meßbereich kann durch Variation von $i_{\mathrm{s}}$ bequem eingestellt werden.

Herrn Prof. Dr. K. M. Косн möchte ich für das fördernde Interesse an dieser Arbeit danken. Herr Dr. F. Kuhrt, Siemens-Schuckertwerke Nürnberg, war so freundlich, uns den HaLL-Multiplikator zur Verfügung $\mathrm{zu}$ stellen. 